\title{
Trajectory tracking control of agricultural vehicles based on disturbance test
}

\author{
Zhengduo Liu ${ }^{1,2}$, Wenxiu Zheng ${ }^{1,2}$, Neng Wang ${ }^{3}$, Zhaoqin Lyu ${ }^{1,2}$, Wanzhi Zhang ${ }^{1,2 *}$ \\ (1. College of Mechanical and Electronic Engineering, Shandong Agricultural University, Taian 271018, Shandong, China; \\ 2. Shandong Provincial Key Laboratory of Horticultural Machinery and Equipment, Taian 271018, Shandong, China; \\ 3. College of Physics and Electronic Science, Hunan University of Science and Technology, Xiangtan 411201, Hunan, China)
}

\begin{abstract}
To improve the trajectory tracking robust stability of agricultural vehicles, a path tracking control method combined with the characteristics of agricultural vehicles and nonlinear model predictive control was presented. Through the proposed method, the path tracking problem can be divided into two problems with speed and steering angle constraints: the trajectory planning problem, and the trajectory tracking optimization problem. Firstly, the nonlinear kinematics model of the agricultural vehicle was discretized, then the derived model was inferred and regarded as the prediction function plant for the designed controller. Second, the objective function characterizing the tracking performance was put forward based on system variables and control inputs. Therefore, the objective function optimization problem, based on the proposed prediction equation plant, can be regarded as the nonlinear constrained optimization problem. What's more, to enhance the robust stability of the system, a real-time feedback and rolling adjustment strategy was adopted to achieve optimal control. To validate the theoretical analysis before, the Matlab simulation was performed to investigate the path tracking performance. The simulation results show that the controller can realize effective trajectory tracking and possesses good robust stability. Meanwhile, the corresponding experiments were conducted. When the test vehicle tracked the reference track with a speed of $3 \mathrm{~m} / \mathrm{s}$, the maximum lateral deviation was $13.36 \mathrm{~cm}$, and the maximum longitudinal deviation was $34.61 \mathrm{~cm}$. When the added horizontal deviation disturbance $Y r$ was less than $1.5 \mathrm{~m}$, the controller could adjust the vehicle quickly to make the test car return to the reference track and continue to drive. Finally, to better highlight the controller proposed in this paper, a comparison experiment with a linear model predictive controller was performed. Compared to the conventional linear model predictive controller, the horizontal off-track distance reduced by $36.8 \%$ and the longitudinal deviation reduced by $32.98 \%$ when performing circular path tracking at a speed of $3 \mathrm{~m} / \mathrm{s}$.
\end{abstract}

Keywords: path tracking, nonlinearity, controller, robustness

DOI: $10.25165 /$ j.ijabe.20201302.4506

Citation: Liu Z D, Zheng W X, Wang N, Lyu Z Q, Zhang W Z. Trajectory tracking control of agricultural vehicles based on disturbance test. Int J Agric \& Biol Eng, 2020; 13(2): 138-145.

\section{Introduction}

The trajectory tracking of agricultural machinery is the key technology to realize the automation and intelligence of agricultural machinery. The difficulty of path tracking lies in the complicated working environment and the agricultural machinery can difficulty exercise sound judgment. At present, the autonomous driving of small agricultural transport vehicles has made important progress in the indoor field ${ }^{[1-8]}$. However, affected by natural environment (slope, ground obstacle, surface condition, climate condition) and other factors, outdoor agricultural transport vehicles are difficult to ensure the accuracy and robustness of trajectory tracking ${ }^{[9]}$.

With the development of control theory, domestic and foreign scholars have made some relevant studies on the trajectory tracking

Received date: 2018-07-11 Accepted date: 2019-12-25

Biographies: Zhengduo Liu, PhD candidate, research interests: intelligent agricultural machinery, Email: 1362825417@qq.com; Wenxiu Zheng, PhD, research interests: intelligent agricultural machinery, Email: wenxiu9013@163.com; Neng Wang, Bachelor candidate, research interests: computer visual and Deep Learning, Email: senbon.nagi@outlook.com; Zhaoqin Lyu, PhD, Professor, research interests: potato machinery, intelligent agricultural machinery. Email: lzqsdau2003@126.com.

*Corresponding author: Wangzhi Zhang, $\mathrm{PhD}$, Lecture, research interests: potato machinery, intelligence and unmanned agricultural machinery. College of Mechanical and Electronic Engineering, Shandong Agricultural University, Taian 271018, China. Email: zhangwanzhi@163.com. of agricultural vehicles ${ }^{[10-12]}$. Machine vision can collect a lot of path information. It can identify crop rows with image processing technology and then determine navigation datum to realize navigation of agricultural machinery. In response to the problem of autonomous walking of agricultural robots in the field, $\operatorname{Sun}^{[13]}$ designed modern seedling recognition and path tracking control systems using modern image processing technology, which can plan walking paths and control the tracking paths of agricultural robots. Wu et al. ${ }^{[14]}$ proposed a dual-view-window path tracking method for AGV station identification and path tracking. Experiments showed that the method improved the real-time and robustness of path feature recognition. In the environment, the average feature recognition accuracy is $99.5 \%$. In view of the navigation problem of agricultural transport vehicles in arched sheds, Li et al. ${ }^{[15]}$ used the chroma method to segment crops and roads to improve the accuracy and real-time performance of path tracking. The average deviation of recognition is $2.4 \mathrm{~cm}$ and the detection speed is $240 \mathrm{~ms} / \mathrm{f}$. Machine vision is greatly affected by lighting in outdoor conditions. Meanwhile, due to a large number of images need to be processed, the real-time is poor. With the development of GPS technology, RTK-DGPS high-precision automatic navigation system has been widely used in practical agricultural production ${ }^{[16-18]}$. Luo et al. ${ }^{[19]}$ adopted the RTK-DGPS positioning technology to design the automatic navigation system of the Dongfanghong X-804 tractor and verified the accuracy and reliability of the control system through field 
experiments. Erkan et al. ${ }^{[20]}$ designed the fuzzy control path tracking controller based on the nonlinear least square method and developed the navigation control system based on GNSS, but it had large tracking error in experiment. This was because the tree branches and leaves occlusion signal receiver, so that the signal receiver cannot receive the satellite signal stably, resulting in a large deviation. $\mathrm{Li}$ et al. ${ }^{[21,22]}$ designed an automatic navigation system based on the combination of RTK-DGPS positioning and dual closed-loop steering control. The method for improving the precision of navigation control of agricultural machinery was studied. Experiments showed that the average value of path tracking error does not exceed $0.019 \mathrm{~m}$ and the standard deviation does not exceed $0.041 \mathrm{~m}$.

Thus it can be seen that due to the complexity of the working environment (branches and leaves of the trees keep out signal, high temperature, dust, ground inequality), agricultural machinery relies on external positioning makes the positioning inaccurate and the robustness poor ${ }^{[23-25]}$.

To solve the above problems, this paper combines the model predictive control and agricultural transport vehicle to design navigation controller to realize the trajectory tracking of agricultural vehicles. Model predictive control is widely used in

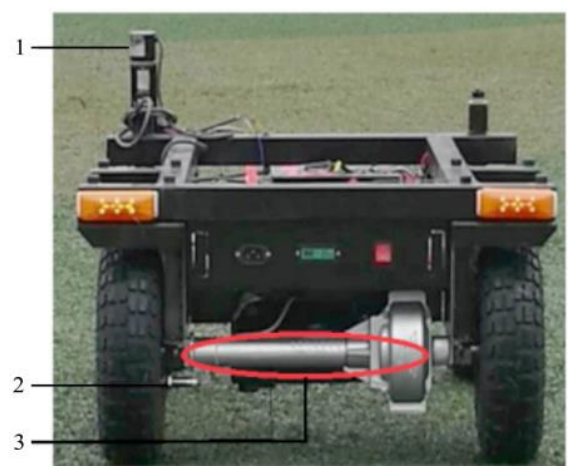

a. Overall structure of test car automatic vehicle navigation ${ }^{[26,27]}$, active front wheel steering ${ }^{[28,29]}$ and other aspects. It can make up for the uncertainty caused by model mismatch, time change and interference. Felix et al. ${ }^{[30]}$ based on the analytical model predictive control theory, combined with the nonlinear model predictive control, designed a kind of ship positioning control system. It can make the ship move rapidly to the reference position. Velenis et al. ${ }^{[31]}$ designed a trajectory tracking controller based on a predictive control method taking the frictional limit of the tires of an autonomous vehicle into consideration. Due to the complexity of the coupled nonlinear model, the calculation time was too long, which did not achieve good results in practical real-time applications. In this paper, a nonlinear model predictive control algorithm is proposed and adopted. Compared with linear model predictive controllers, the model accuracy is improved. Meanwhile, the computational complexity is reduced and the path tracking performance is increased.

\section{Path tracking system structure}

The designed field tracking test vehicle is shown in Figure 1, the related structural parameters in Table 1.

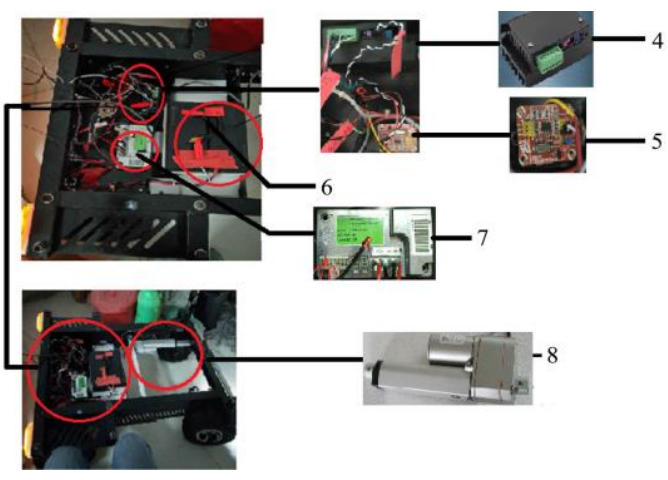

b. Test car electric control section

Figure 1 Test vehicle

Table 1 Main technical indexes of the autonomous vehicle system

\begin{tabular}{lc}
\hline \multicolumn{1}{c}{ Technical index } & Parameters \\
\hline Dimension $\mathrm{L} \times \mathrm{W} \times \mathrm{H} / \mathrm{cm}$ & $120 \times 63 \times 33$ \\
Wheelbase $/ \mathrm{cm}$ & 100 \\
Maximum speed $/ \mathrm{m} \cdot \mathrm{s}^{-1}$ & 3.2 \\
Weight $/ \mathrm{kg}$ & 50 \\
Control method & $\mathrm{PWM}$ \\
Tire radius $/ \mathrm{cm}$ & 15 \\
\hline
\end{tabular}

The main parts include a path information acquisition module, an E6B2-CCWZ3E type angle encoder sensor with a measurement error of $\pm 0.3^{\circ}$, a JK5002D Hall speed sensor with speed error of $\pm 0.05 \mathrm{~m} / \mathrm{s}$, a control execution module, and a path information processing module.

The front-wheel steering driver is the ASMT-01 series high-power linear servo controller. The front-wheel steering actuator is a 42BYGH47 step with a stroke of $80 \mathrm{~mm}$ and a maximum speed of $29 \mathrm{~mm} / \mathrm{s}$. The rear-wheel drive is a CM2010 brushless motor controller. The rear-wheel actuator is a DMW86 brushless DC motor with a rated speed of $3200 \mathrm{r} / \mathrm{min}$ and a rated power of $1000 \mathrm{~W}$.

Path information processing module is a navigation controller comprised of a STM32F103ZET6 type microcontroller. The main task is to collect the information from the speed sensor and the front-wheel angle sensor of the tested vehicle. The information is sent to host computer via WIFI and processed by Matlab software. Its structure and function are shown in Figure 2.

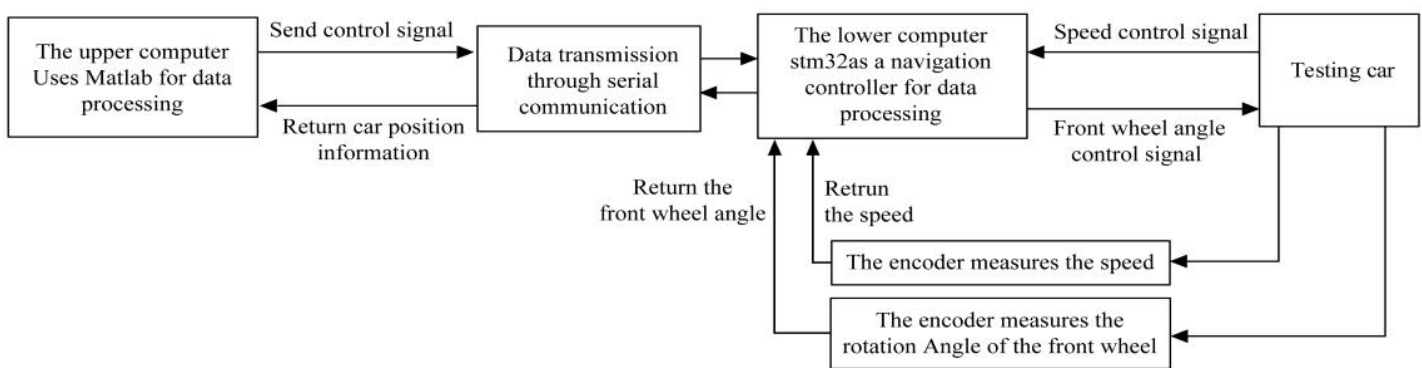

Figure 2 Flow of path tracking 


\section{Kinematic model of agricultural vehicles}

The kinematics analysis of agricultural vehicles is carried out and the kinematics model shown in Figure 3 is established in the plane coordinate system. The front wheel of the model turns and rear wheel drive. In the process of work, the steering wheel and the driving wheel adjust the angle and speed by adjusting the voltage. In the whole kinematics analysis, the agricultural vehicle is regarded as a rigid body running on horizontal plane. In order to determine the agricultural vehicle's position and attitude in the whole trajectory, the navigation coordinate is set up. The center point of the test car's rear axle was selected as a reference point to define the position and position information of the car. Pose information is defined as $(x, y, \varphi)$. In which, $x, y$ are the axial coordinates of the vehicle's rear axle $(\mathrm{m}) ; \varphi$ is the heading angle (rad); $\delta f$ is the front wheel steering angle (rad); $v$ is the center speed of the rear axle $(\mathrm{m} / \mathrm{s}) ; v_{f}$ is the front axle center speed $(\mathrm{m} / \mathrm{s})$; $l$ is the wheelbase (m). Regardless of the roll, pitch, side slip of the agricultural vehicles, the low-speed agricultural vehicles can be reduced to a two-degree-of-freedom kinematic math model ${ }^{[32-35]}$.

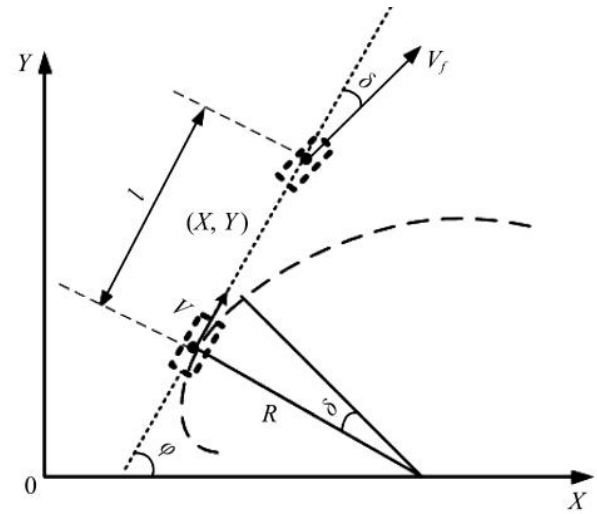

Figure 3 Vehicle kinematic model

The movement of agricultural vehicles belongs to rigid body movement and its kinematics equation is easy to obtain:

$$
\left[\begin{array}{c}
\dot{x}_{r} \\
\dot{y}_{r} \\
\dot{\varphi}
\end{array}\right]=\left[\begin{array}{c}
\cos \varphi \\
\sin \varphi \\
\frac{\tan \delta}{l}
\end{array}\right] v
$$

where, $x$ is global abscissa of agricultural vehicles, $\mathrm{m}$; $y$ is global ordinate of agricultural vehicles, $\mathrm{m}$; The kinematic model shown in Equation (1) can be expressed in the state space model with input variable $u=\left(v, \delta_{f}\right)$ and state variables $\xi=(x, y, \varphi)$ :

$$
\left\{\begin{array}{l}
\dot{\boldsymbol{\xi}}=f(\boldsymbol{\xi}, \boldsymbol{u}) \\
\boldsymbol{\eta}=\boldsymbol{h} * \boldsymbol{\xi}
\end{array}\right.
$$

The output variable $\boldsymbol{\eta}=[x, y, \varphi]^{T}, \boldsymbol{h}=\left[\begin{array}{lll}1 & 0 & 0 \\ 0 & 1 & 0 \\ 0 & 0 & 1\end{array}\right]$.

\section{Design of nonlinear model predictive controller}

\subsection{Prediction model}

In the nonlinear model predictive control, the traditional algorithm has the problem of large computation and slow convergence. This paper use an explicit iterative equation by using Euler method to solve the discretization of the kinematics model. So the convergence rate of the system is accelerated by decreasing the computation .

$$
\left\{\begin{array}{l}
x(k+1)=x(k)+T v \cos (\varphi(k+1)) \\
y(k+1)=y(k)+T v \sin (\varphi(k+1)) \\
\varphi(k+1)=\varphi(k)+\frac{T v \tan \delta}{l}
\end{array}\right.
$$

where, $k$ is a discrete time variable; $(x(k), y(k))$ is the axial of agricultural vehicles at moment $k, \mathrm{~m} ; \varphi(k)$ is the heading angle at moment $k, \operatorname{rad} ; v_{k}$ is the speed of agricultural vehicles at moment $k$, $\mathrm{m} / \mathrm{s} ; \delta_{f k}$ is the front wheel Angle of the agricultural vehicle at moment $k$, rad;

According to this mathematic model, it is necessary to know the initial pose information $(x(k), y(k), \varphi(k))$ of the controlled system at a certain time and the control input sequence $\left(v_{1}, \delta_{f 1}\right),\left(v_{2}, \delta_{f 2}\right)$ to predict the output sequence of the system in the predicted $k+1$ time domain. Equation (3) is sorted into the form of output function:

$$
\begin{aligned}
& \boldsymbol{\eta}_{p}(t+j \mid t)=\boldsymbol{F}_{j}\left(\xi(t \mid t), u(t \mid t), \ldots, u\left(t+N_{\mathrm{c}}-1 \mid t\right)\right) \\
& \left(j=1,2, \ldots, N_{p}\right)
\end{aligned}
$$

where, $N_{p}$ is predicted time domain; $N_{c}$ is controlled time domain.

$$
\begin{aligned}
& N_{\mathrm{c}}=\left\{\begin{array}{l}
1, j=1 \\
2, j>1
\end{array}\right. \\
& \boldsymbol{F}_{j}(\cdot)=\left[\begin{array}{l}
\sum_{i=1}^{j} x(i \mid t)+T v \sum_{i=1}^{j} \cos (\varphi(i+1 \mid t)) \\
\sum_{i=1}^{j} y(i \mid t)+T v \sum_{i=1}^{j} \sin (\varphi(i+1 \mid t)) \\
\sum_{i=1}^{j} \varphi(i \mid t)+\frac{T v}{l} \tan \delta_{f}
\end{array}\right]
\end{aligned}
$$

To reduce the computational complexity and enhance the real-time performance of the control system. This paper $N_{c}=2$, thus, the prediction model of nonlinear model predictive controller is obtained:

$$
\Gamma_{p}(t)=\left[\begin{array}{c}
\boldsymbol{\eta}_{\mathrm{p}}(t+1 \mid t) \\
\boldsymbol{\eta}_{\mathrm{p}}(t+2 \mid t) \\
\vdots \\
\boldsymbol{\eta}_{\mathrm{p}}(t+j \mid t) \\
\vdots \\
\boldsymbol{\eta}_{\mathrm{p}}\left(t+N_{p} \mid t\right)
\end{array}\right]=\left(\begin{array}{c}
\boldsymbol{F}_{1}(\cdot) \\
\boldsymbol{F}_{2}(\cdot) \\
\vdots \\
\boldsymbol{F}_{j}(\cdot) \\
\vdots \\
\boldsymbol{F}_{N_{p}}(\cdot)
\end{array}\right), j=1,2, \ldots, N_{p}
$$

\subsection{Closed-loop correction for nonlinear models}

In order to improve the robust performance of the control system, closed-loop correction of the prediction model is coined based on the error prediction and real-time feedback.

$$
\begin{aligned}
& \boldsymbol{e}(t+i-1 \mid t)=\Gamma_{r}(t+i-1 \mid t)-\Gamma_{p}(t+i-1 \mid t) \\
& i=1,2, \ldots, N_{p}
\end{aligned}
$$

where, $\boldsymbol{e}(t+i-1 \mid t)$ is the error of the system at $t+i-1$ based on the real-time information measurement at $t ; \boldsymbol{\Gamma}_{r}(t+i-1 \mid t)$ is the system output at $t+i-1$ based on the real-time information measurement at $t$ $\Gamma_{p}(t+i-1 \mid t)$ is the output at $t+i-1$ based on kinematics model of the agricultural vehicle at $t$.

$$
\begin{aligned}
& \boldsymbol{\Gamma}_{r}(t)=\left[\boldsymbol{\eta}_{r}(t+1 \mid t) \ldots \boldsymbol{\eta}_{r}(t+i \mid t) \ldots \boldsymbol{\eta}_{r}\left(t+N_{p} \mid t\right)\right]^{T}, i=1,2, \ldots, N_{p} \\
& \boldsymbol{\eta}_{r}(t+i-1 \mid t)=\left[\begin{array}{l}
x_{r}(t+i-1 \mid t) \\
y_{r}(t+i-1 \mid t) \\
\varphi_{r}(t+i-1 \mid t)
\end{array}\right]=\left[\begin{array}{l}
X_{r}(t)+T v_{c} \cos (\varphi(i+1)) \\
Y_{r}(t)+T v_{c} \sin (\varphi(i+1)) \\
\varphi_{r}(t)+T \frac{\left(v_{c} \tan \delta_{c}\right)}{l}
\end{array}\right] \\
& i=1,2, \ldots, N_{p} \\
& c=\left\{\begin{array}{l}
=1, i=1 \\
=2, i>1
\end{array}\right.
\end{aligned}
$$


$X_{r}(t), Y_{r}(t), \varphi_{r}(t)$ is the real-time feedback of the car's actual state of motion; $v_{c}, \delta_{f c}$ is the system real-time control variable.

Assume that $f(0,0)=0$ is the stable operating point of the system. The objective function is set up to solve the problem and the method of soft constraint is proposed to prevent the system from appearing unfeasible. For any predicted time domain, the objective function $J(\cdot, \cdot)$ is shown in Equation (8).

$$
\boldsymbol{J}=\sum_{i=1}^{N_{P}} \Delta \boldsymbol{\Gamma}(i \mid t) \boldsymbol{Q} \Delta \boldsymbol{\Gamma}^{\mathrm{T}}(i \mid t)+\sum_{i=1}^{N_{c}-1} \Delta \boldsymbol{u}(i \mid t) \boldsymbol{R} \Delta \boldsymbol{u}^{\mathrm{T}}(i \mid t)+\rho \varepsilon^{2}
$$

where, $\Delta u$ is the input control variable, which is used to limit the variation of the control amount; $\Delta \boldsymbol{\Gamma}$ is the lateral deviation, which is used to limit and minimize the lateral deviation; the relaxation factor $\varepsilon^{2}$ is used to control the change of the variable each iteration, which mainly affects the convergence speed and convergence of the iteration In some cases, the smaller the relaxation factor, the smaller the change between two iterations.

$$
\begin{aligned}
\Delta \Gamma(i \mid t) & =\Gamma_{r}(i \mid t)-\Gamma_{p}(i \mid t) \\
& =\left[\begin{array}{llll}
\boldsymbol{e}(t+1 \mid t) & \boldsymbol{e}(t+1 \mid t) & \cdots & \boldsymbol{e}(t+i \mid t)
\end{array}\right]^{\mathrm{T}}
\end{aligned}
$$

The path tracking problem is transformed to solve the constraint problem of nonlinear quadratic form by setting the value range of state quantity and control quantity. Both the objective function and the constraint function are continuous, and the gradient is continuous in Equation (8). Therefore, the objective optimization problem is can be solved based on quadratic programming of the recursive sequence. Taking the nonlinear boundary constraints into account, the optimization problem can be summarized as $\min$

$$
\boldsymbol{J}=\sum_{i=1}^{N_{P}} \Delta \boldsymbol{\Gamma}(i \mid t) \boldsymbol{Q} \Delta \boldsymbol{\Gamma}(i \mid t)^{\mathrm{T}}+\sum_{i=1}^{N c-1} \Delta \boldsymbol{u}(i \mid t) \boldsymbol{R} \Delta \boldsymbol{u}(i \mid t)^{\mathrm{T}}+\rho \varepsilon^{2}
$$

s.t.

$$
\begin{aligned}
& \Gamma_{\min } \leq \boldsymbol{\Gamma}(i \mid t) \leq \Gamma_{\max } \\
& \Delta \boldsymbol{\Gamma}_{\min } \leq \Delta \boldsymbol{\Gamma}(i \mid t) \leq \Delta \boldsymbol{\Gamma}_{\max } \\
& \boldsymbol{u}_{\min } \leq \boldsymbol{u}(i \mid t) \leq \boldsymbol{u}_{\max }\left(k=0,1, \cdots, N_{c}-1\right) \\
& \Delta \boldsymbol{u}_{\min } \leq \Delta \boldsymbol{u}(i \mid t) \leq \Delta \boldsymbol{u}_{\max }\left(k=0,1, \cdots, N_{c}-1\right)
\end{aligned}
$$

\section{Simulation result and analysis}

\subsection{Test site}

Figure 4 is the experiment place in the plum forest of Shandong Agricultural University and Figure 5 shows the reference trajectory of the agricultural driverless vehicle traveling from point $O$ to point $G$.

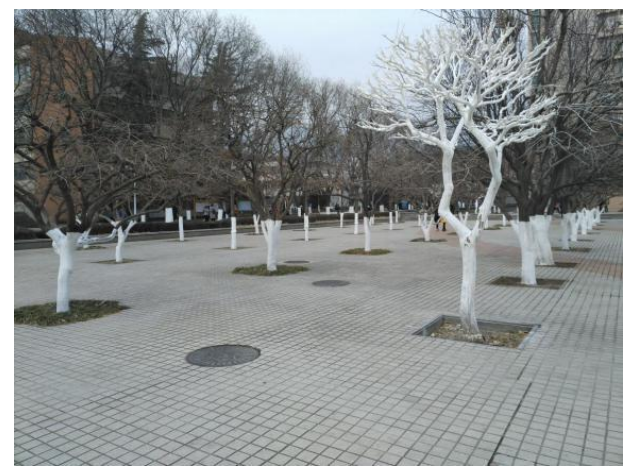

Figure 4 Forest of Shandong reference path

The agricultural vehicle starts from the $O$ point to $G$ point for the end. Where $A\left(X_{p}, 0\right), B(20,1), C(22,5), D(10.3,5), E(0,5)$, $F(-2,9), G(20,9)$. The tracking performance can be evaluated by the parameters observed at these points. Point $A$ is defined as the maximum overshoot point at the transient response during vehicle path tracking. Point $D$ is defined as the disturbance test point and additional disturbance is added to test the robustness of the nonlinear model predictive controller. The model predictive controller is designed in Matlab platform. The simulation analysis is carried out from these four aspects: forward speed $v$, sampling period $T$, lateral deviation disturbance $Y_{r}$ and front wheel corner disturbance $\delta_{f r}$.

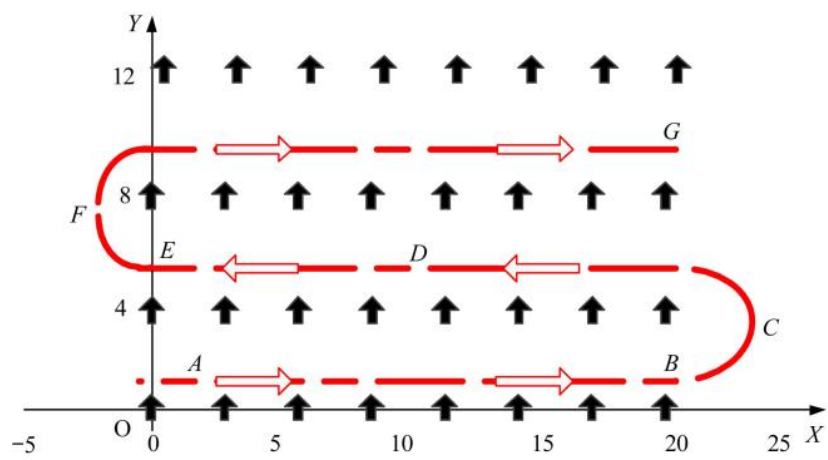

Figure 5 Experiment path trajectory

The controller parameters are set according to the path information (the reference path coordinate range and the parameter range of the front-wheel steering angle when the car is turning) and the target speed:

$l=1 \mathrm{~m}$;

$\boldsymbol{Q}=\operatorname{diag}(100,100,100), R=100$;

$\boldsymbol{u}_{\min }=\left[\begin{array}{ll}-3.2 \mathrm{~m} / \mathrm{s} & -0.5 \mathrm{rad}\end{array}\right]^{\mathrm{T}}, \boldsymbol{u}_{\max }=\left[\begin{array}{ll}3.2 \mathrm{~m} / \mathrm{s} & 0.5 \mathrm{rad}\end{array}\right]^{\mathrm{T}}$;

$\Delta \boldsymbol{u}_{\min }=\left[\begin{array}{ll}-0.05 \mathrm{~m} / \mathrm{s} & -0.47 \mathrm{rad}\end{array}\right]^{\mathrm{T}}, \Delta \boldsymbol{u}_{\max }=\left[\begin{array}{ll}0.05 \mathrm{~m} / \mathrm{s} & 0.47 \mathrm{rad}\end{array}\right]^{\mathrm{T}}$;

$\Gamma_{\min }=\left[\begin{array}{lll}-1 \mathrm{~m} & -1 \mathrm{~m} & -0.5 \mathrm{rad}\end{array}\right]^{\mathrm{T}}, \boldsymbol{\Gamma}_{\max }=\left[\begin{array}{lll}22 \mathrm{~m} & 12 \mathrm{~m} & 0.5 \mathrm{rad}\end{array}\right]^{\mathrm{T}}$;

$\Delta \boldsymbol{\Gamma}_{\min }=\left[\begin{array}{lll}-0.05 \mathrm{~m} & -0.05 \mathrm{~m} & -0.0082 \mathrm{rad}\end{array}\right]^{\mathrm{T}}$,

$\Delta \boldsymbol{\Gamma}_{\max }=\left[\begin{array}{lll}0.05 \mathrm{~m} & 0.05 \mathrm{~m} & 0.0082 \mathrm{rad}\end{array}\right]^{\mathrm{T}}$

\subsection{Impact of speed on path tracking}

The initial control parameters are set as $N_{p}=45, N_{c}=2, T=0.03 \mathrm{~s}$, $v=1 \mathrm{~m} / \mathrm{s}, 2 \mathrm{~m} / \mathrm{s}, 3 \mathrm{~m} / \mathrm{s}$. In the navigation coordinate system, the trajectory tracking results are shown as Figure 6.

Figure 6 shows that the car tracks the reference path well at the speed of $3 \mathrm{~m} / \mathrm{s}$ or less. It can be seen from Figures $6 \mathrm{~b}$ and $6 \mathrm{c}$ that the overshoot and adjustment time reach a maximum at the speed of $1 \mathrm{~m} / \mathrm{s}$ in transient response. The lateral tracking deviation reaches $7.51 \mathrm{~cm}$ and the longitudinal tracking deviation reaches $-5.93 \mathrm{~cm}$. The adjustment time and overshoot decrease with the increase of speed. This is because the regulation of the controller is earlier with faster speed. As a result, the overshoot is decreased.

Figure $6 \mathrm{~d}$, 6e verified the vehicle stability during the path tracking, the increase of speed has a great influence on the driving stability. When the speed is lower than $3 \mathrm{~m} / \mathrm{s}$, the higher the speed, the better the stability in transient response and the worse the stability in the running process. And the designed controller shows better performance during a straight-line path tracking.

\subsection{Impact of sampling time on path tracking}

The initial control parameters are set as $N p=45, N c=2, T=$ $0.01 \mathrm{~s}, 0.02 \mathrm{~s}, 0.03 \mathrm{~s}, v=3 \mathrm{~m} / \mathrm{s}$. In the navigation coordinate system, the trajectory tracking results are shown as Figure 7.

The control accuracy of nonlinear model prediction depends on the accuracy of the nonlinear prediction model. In this paper, the Euler method is used to solve the prediction model. Therefore, the accuracy of the prediction model is directly related to the 
sampling period T. It can be seen from Figure 7a, $7 \mathrm{~b}$ that as the sampling period becoming larger, the variation of horizontal tracking deviation and vertical tracking deviation gradually becomes larger and the accuracy decreases.

Regarding the tracking stability, it is shown in Figure $7 \mathrm{c}$ and Figure $7 \mathrm{~d}$ that the front wheel angle and speed are more stable with the longer sampling period in transient response. However, from nodes $\mathrm{B}, \mathrm{C}, \mathrm{E}, \mathrm{F}$ on tracking path, the stability deteriorates when the sampling period becomes longer. This is due to a change in the trajectory equation, which requires the controller to re-predict

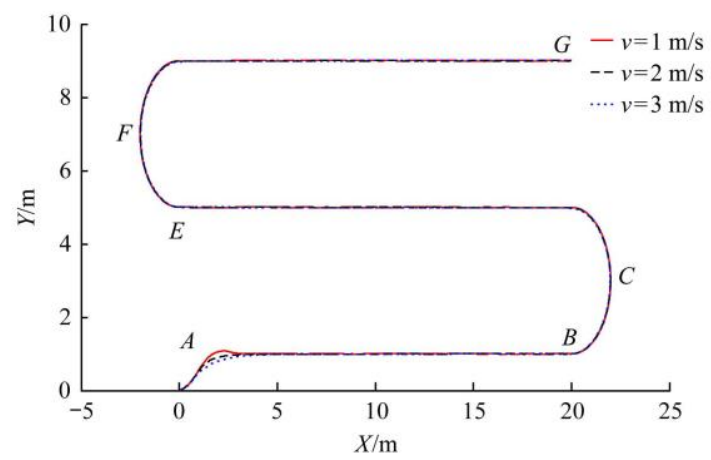

a. Path tracking

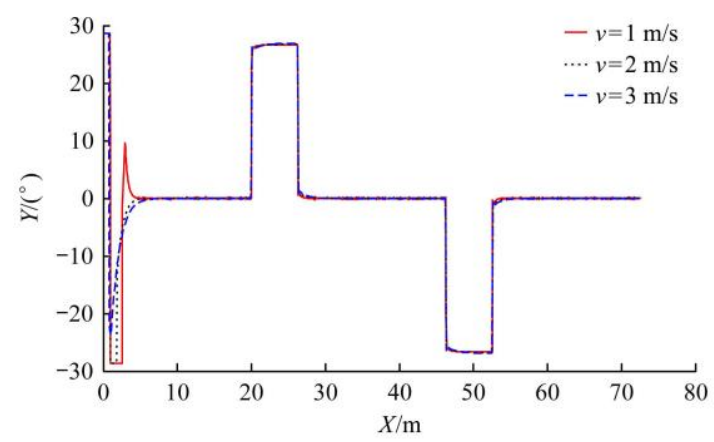

c. Tracking error in longitudinal
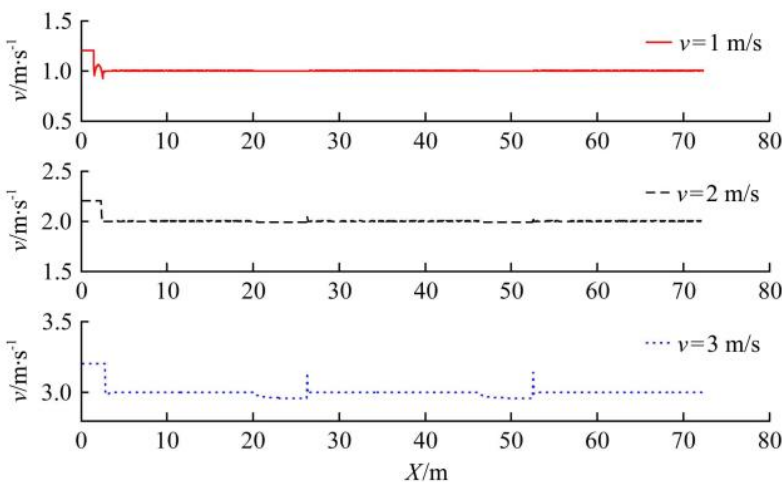

e. Velocity

Figure 6 Path tracking performance under different speed

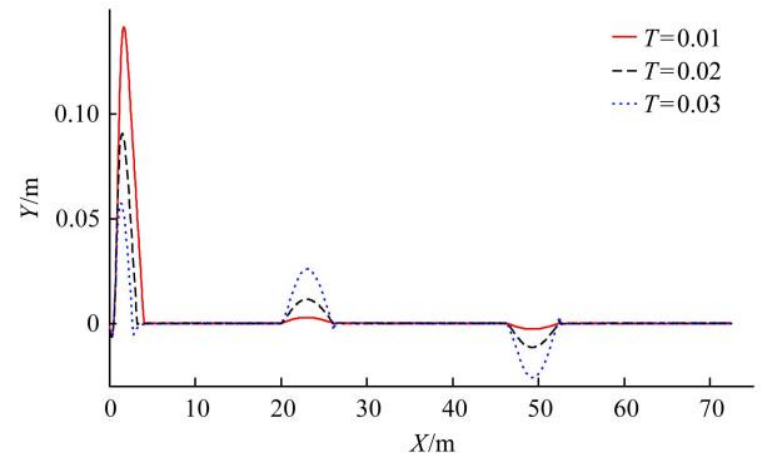

a. Tracking error in lateral direction the system parameters to get accommodated to the new path. In conclusion, an increase of the sampling period under the simulation condition will reduce the accuracy of the path tracking, at the same time it will improve the stability in the initial stage of driving and reduce the stability in the driving process.

\subsection{Impact of lateral noise on path tracking}

The initial control parameters are set as $N p=45, N c=2, T=$ $0.03 \mathrm{~s}, v=3 \mathrm{~m} / \mathrm{s}, Y r=0.5 \mathrm{~m}, 1 \mathrm{~m}, 1.5 \mathrm{~m}$. In the navigation coordinate system, the trajectory tracking results are shown as Figure 8.

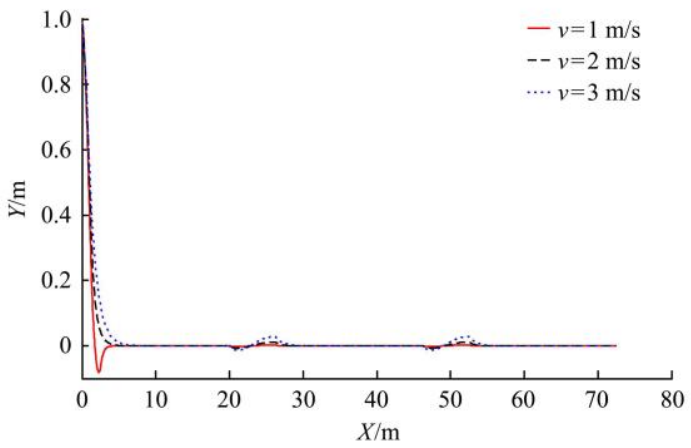

b. Tracking error in lateral direction

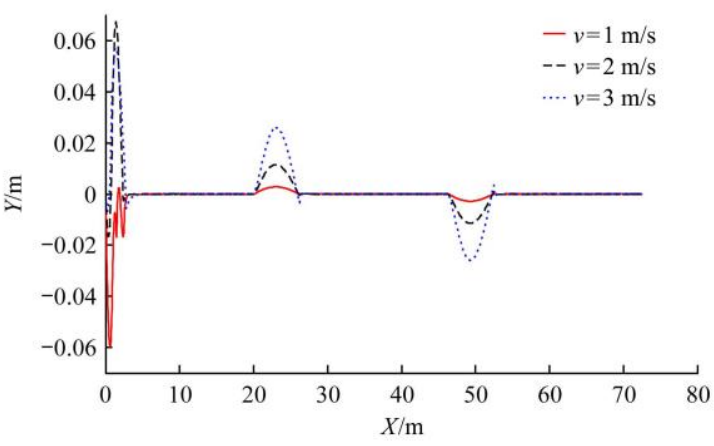

d. Front wheel angle direction

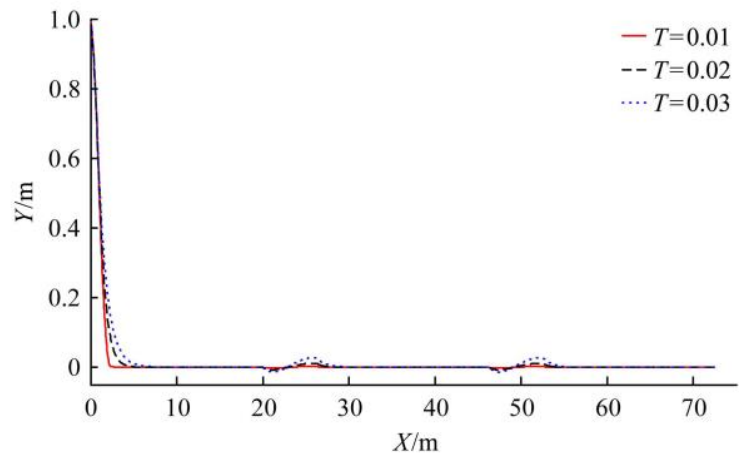

b. Tracking error in longitudinal 


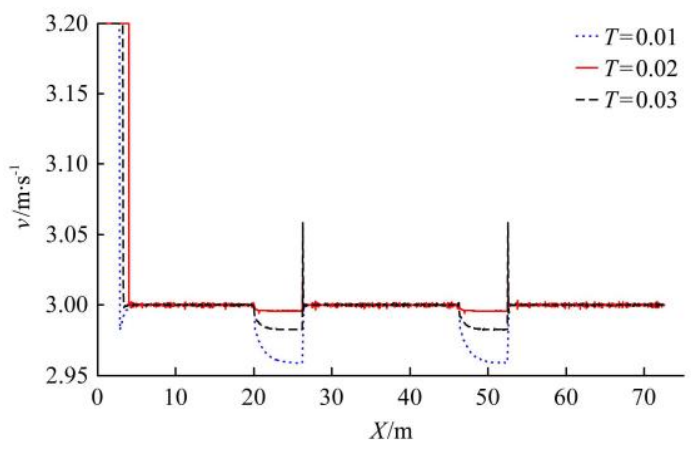

c. Front wheel angle direction

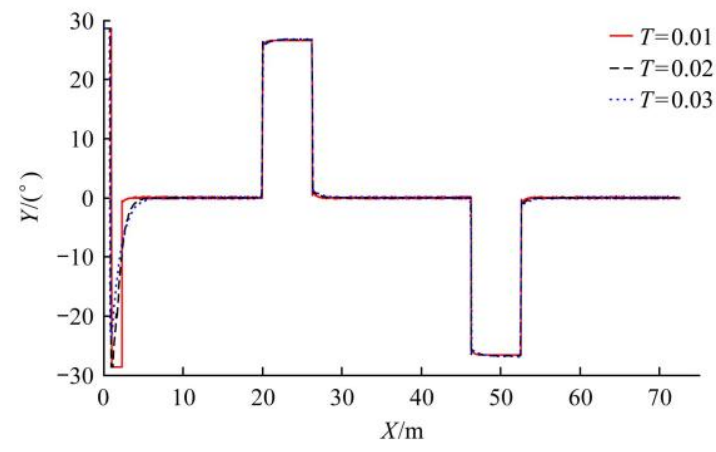

d. Velocity

Figure 7 Effect of sampling period on path tracking

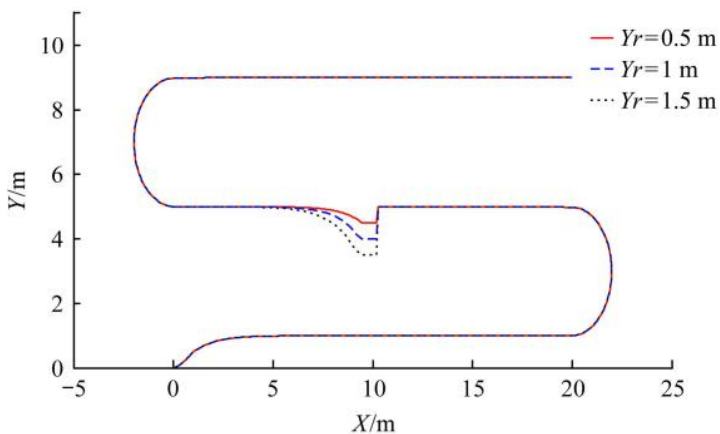

a. Path tracking

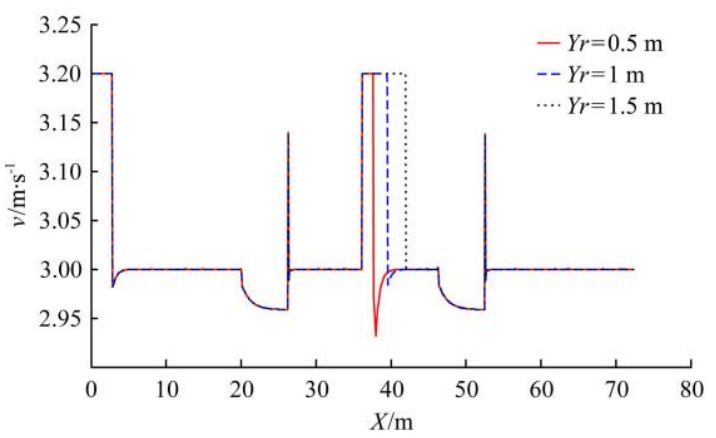

c. Tracking error in longitudinal

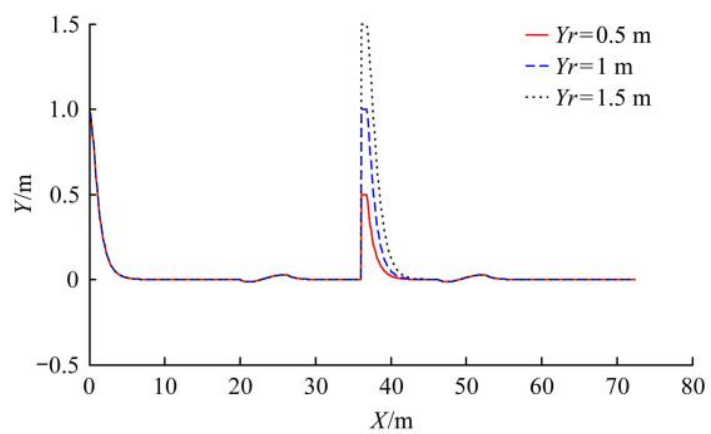

b. Tracking error in lateral direction

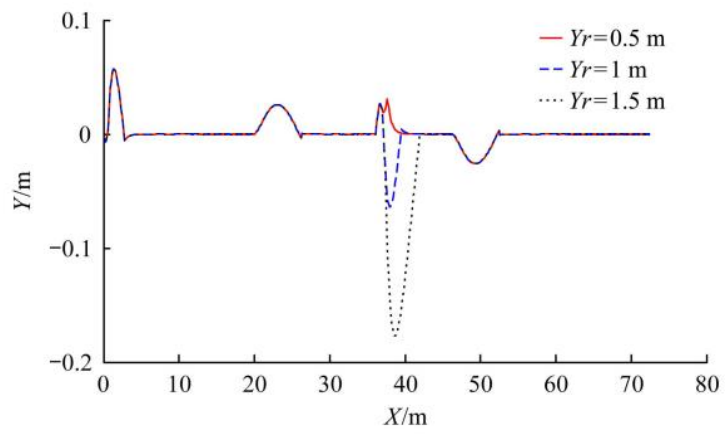

d. Front wheel angle direction

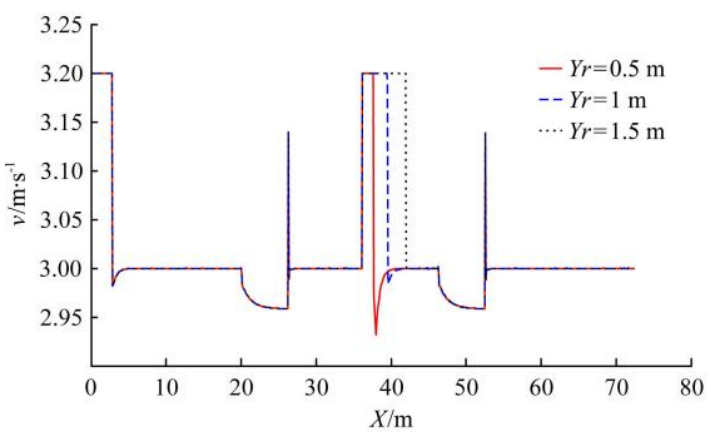

e. Velocity

Figure 8 Effect of lateral disturbance on path tracking

When unmanned vehicles deliver fruits, the vehicles need to perform the horizontal movement. The horizontal movement is taken as a disturbance for the system. The lateral deviation disturbance is injected at node D. As the disturbance increases, the tracking deviation and the adjustment time also increase. From Figure 8d and 8e, when the disturbance occurs, the controller will quickly respond to adjust the front wheel angle and speed so that the vehicle can back to the reference path as soon as possible.

\section{Experiment and analysis}

\subsection{Path tracking test}

The experiment takes place at Shandong Agricultural University. Test site has been shown as Figure 9.

The initial control parameters are set as $N p=45, N c=2, T=0.03 \mathrm{~s}$, $v=3 \mathrm{~m} / \mathrm{s}$. To verify the robustness of the designed controller, lateral deviation disturbance $Y r=0.5 \mathrm{~m}, 1 \mathrm{~m}, 1.5 \mathrm{~m}$ to node D. The experiment result is shown in Figure 10.

It can be seen from Figure 10 that the off-track deviation of the straight line section is small. When tracking the circular section, the off-track deviation is large. The specific data as shown in Table 2.

The results illustrate that the test car can effectively track the 
reference path with the nonlinear model predictive controller. The deviations mainly appear in the locations where the path curves change and the locations where the perturbations are added such as nodes C, D and F. As is shown in Table 2, the maximum lateral deviation is $13.36 \mathrm{~cm}$ and the maximum longitudinal deviation is $23.41 \mathrm{~cm}$. There are mainly two reasons for the deviation. On one hand, experimental constraints: linkages between the various trailers, the delay of steering actuator, data transmission, and data processing. On the other hand, the reference trajectory changes occur at the end of the straight line path, where the car already has

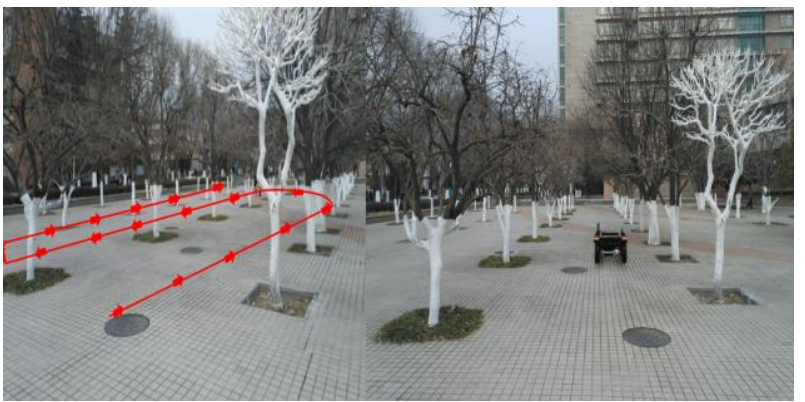

Figure 9 Tracking experiment

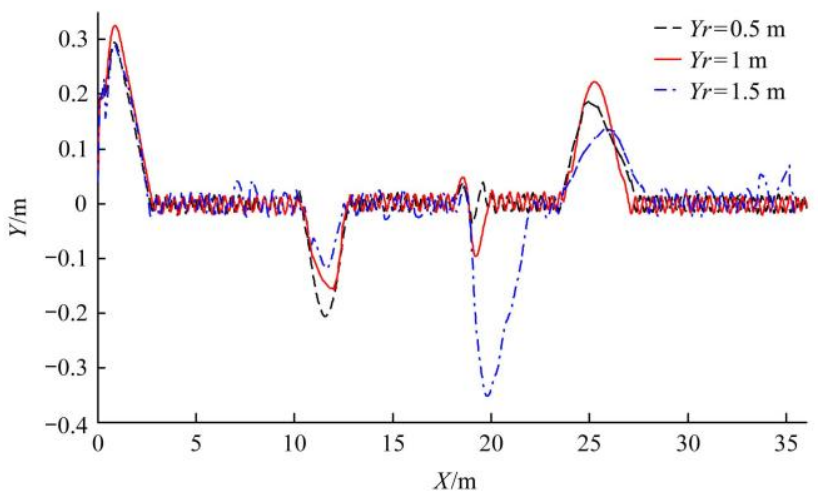

a. Path tracking

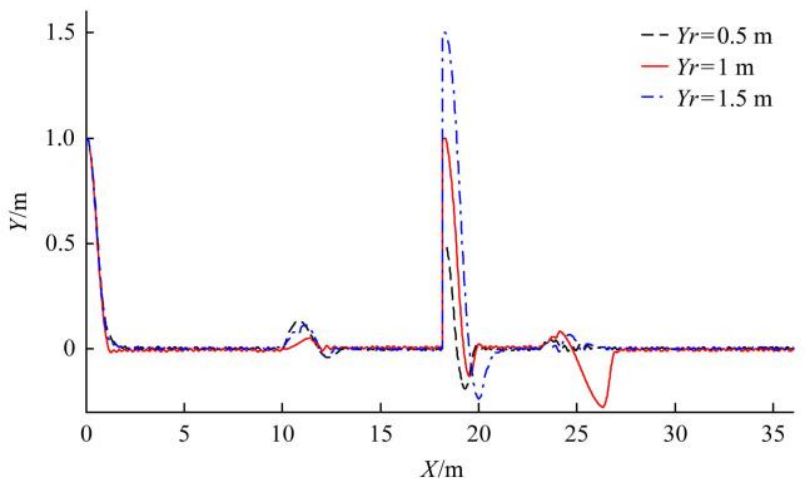

b. Tracking error in lateral direction

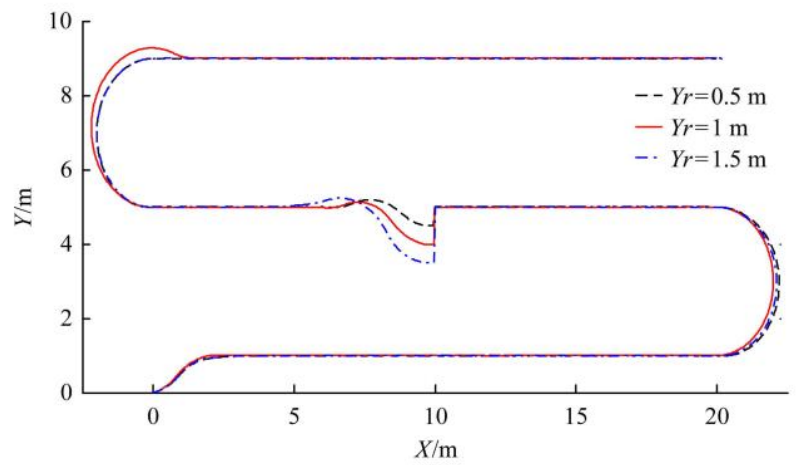

c. Tracking error in longitudinal

Figure 10 Experiment result some cumulative deviation from the previous maneuvering process. The controller performs well over the straight section, which can be seen from the nodes $\mathrm{B}, \mathrm{E}$ and $\mathrm{G}$ that the maximum lateral deviation is $9.01 \mathrm{~cm}$ and the maximum longitudinal deviation is $12.94 \mathrm{~cm}$. It can be seen that the controller can meet the requirements of precision and stability of track tracking of agricultural vehicles.

\subsection{Comparison with linear model predictive controller}

In order to verify the proposed path-tracking controller, it is compared with the linear model predictive controller method in literature [36] in both the straight path and circular path. The main controller parameters are set $N_{p}=45, N_{c}=2, T=0.03 \mathrm{~s}, v=1 \mathrm{~m} / \mathrm{s}$, $2 \mathrm{~m} / \mathrm{s}$. The experimental results show that the two controllers have similar performance in the tracking accuracy when the velocity is less than $2 \mathrm{~m} / \mathrm{s}$. In the circular path tracking with speed $v=3 \mathrm{~m} / \mathrm{s}$, the test car under the linear model predictive controller showed a larger deviation. The average value of lateral off-track deviation was $12.06 \mathrm{~cm}$ and the average value of longitudinal tracking deviation was $38.04 \mathrm{~cm}$. This is because the nonlinear characteristic of the car become to be prominent. The controller adopting a time-varying linear model as the predictive model demonstrates differences between the estimated trajectory and real trajectory. This will result in a larger tracking deviation. With the proposed controller, the average value of lateral off-track deviation is reduced by $36.8 \%$ and the average of longitudinal track deviation is reduced by $32.98 \%$. Thus, at high speed, the controller has advantages in tracking accuracy.

\section{Conclusions}

(1) In this paper, nonlinear model predictive control of agricultural vehicles is proposed to tackle the low accuracy problem and poor prediction result from the linear model. The Euler method is used to establish the prediction model. Then the predictive control is transformed into quadratic programming problem based on recursive sequence. Finally, the nonlinear model is completed through feedback correction and rolling optimization. The design of the predictive controller is simulated and verified by experiments. It can be seen that the method is of less calculation complexity and high accuracy.

(2) This paper analyzes and tests the robust stability of agricultural vehicles emphatically. The results show that the controller can respond timely when the trajectory equation is changed or the disturbance is added, making the vehicle return to the reference track as soon as possible.

(3) From the field test results, when there is no disturbance and speed less $3 \mathrm{~m} / \mathrm{s}$, the maximum lateral tracking deviation is $13.36 \mathrm{~cm}$ and the maximum longitudinal tracking deviation is $23.41 \mathrm{~cm}$. The automatic navigation of agricultural vehicles is basically realized. When adding maximum lateral deviation $Y_{r}=1.5 \mathrm{~m}$, the controller can quickly adjust the vehicle to go back to the reference trajectory.It is proved that the controller has strong robustness stability while keeping track precision.

\section{Acknowledgements}

This work is supported by Shandong Agricultural Machinery and Equipment Research and Development Innovation Initiative(2018YF020-07, 2017YF002), Modern Agricultural Technology System Innovation Team Post Project in Shandong Province (SDAIT-16-10), the National Key Research Projects (2017 YFD0700705), and the Natural Science Foundation of Shandong Province( ZR2019BC018). 


\section{[References]}

[1] Starcevica N, Thullner C, Bux M, Muller J. 3D path planning for a biomass processing robot via motion simulation. Robotics and Computer-Integrated Manufacturing, 2010; 26(1): 109-118.

[2] Sutoh M, Otsuki M, Wakabayashi S, Hoshino T, Hashimoto T. The right path: comprehensive path planning for lunar exploration rovers. IEEE Robotics \& Automation Magazine, 2015; 22(1): 22-23.

[3] Garcia P L, Garcia A M C, Ribeiro A, Guinea D. An agent of behaviour architecture for unmanned control of a farming vehicle. Computers and Electronics in Agriculture, 2008; 60: 39-48.

[4] Zhao Y S, Gong L, Zhou B, Huang Y X, Niu Q L, Liu C L. Object recognition algorithm of tomato harvesting robot using non-color coding approach. Transactions of the CSAE, 2016; 47(7): 1-7. (in Chinese)

[5] Qian X M, Zhang H, Wang X Y, Wu X. Research on relative positioning technology of mobile robots based on laser scanning matching. Journal of agricultural machinery, 2016; 47(3): 14-21.

[6] Huang Y J, Lee F F. An automatic machine vision-guided grasping system for Phalaenopsis tissue culture plantlets. Computers and Electronics in Agriculture, 2010; 70: 42-51.

[7] Alberto T, Xavier P B, Gonzalo P. A new vision-based approach to differential spraying in precison agriculture. Computers and Electronics, 2010; 70(6): 144-155.

[8] Cen Y, Song C, Xie N, Wang L. Path planning method for mobile robot based on ant colony optimization algorithm. Industrial Electronics and Applications . ICIEA, Iciea, 2008; pp.298-301.

[9] Zhao Y, Yang W. Technological development of agricultural tractor. Transactions of the CSAE, 2010; 41(6): 42-48. (in Chinese)

[10] Wang R G, Zhang H, Wang J. Linear parameter-varying controller design for four-wheel independently actuated electric ground vehicle with active steering systems. IEEE Transactions on Control Systems Technology, 2014; 22(4): 1281-1296.

[11] Enache N M, Mammar S, Netto M, Lusetti B. Driver steering assistance for lane-departure avoidance based on hybrid automata and composite lyapunov function. IEEE Transactions on Intelligent Transportation Systems, 2010; 11(1): 28-39.

[12] Wu S J, Chiang H H, Perng J W. The heterogeneous systems integration design and implementation for lane keeping on a vehicle. IEEE Transactions on Intelligent Transportation Systems, 2008; 9(2): 246-263.

[13] Sun Y Y, Zhang S L, Li W. Guidance lane detection for pesticide spraying robot in cotton fields. Journal of Tsinghua University: Science and Technology, 2007; 47(2): 206-209. (in Chinese)

[14] Wu X, Shen W, Lou P, Wang L. Research on robust feature recognition and accurate path tracking of vision-guided AGV. Journal of Agricultural Machinery, 2016; 47 (7): 48-56.

[15] Li T H, Wu Z H, Lian X K, Hou J L, Shi G Y, Wang Q. Navigation line detection for greenhouse carrier vehicle based on fixed direction camera. Transactions of the CSAE, 2018; 49(S1): 8-13. (in Chinese)

[16] Wei S, Li S C, Zhang M, Ji Y H, Xiang M, Li M Z. Automatic navigation path search and turning control of agricultural machinery based on GNSS. Transactions of the CSAE, 2017; 33(Z1): 70-77. (in Chinese)

[17] Jin B, Li J X, Zhu D K, Guo J, Su B F. GPS/INS navigation based on adaptive finite impulse response-Kalman filter algorithm. Transactions of the CSAE, 2019; 35(3): 75-81. (in Chinese)

[18] Ji C Y, Zhou J. Current situation of navigation technologies for agricultural machinery. Transactions of the CSAE, 2014; 45(9): 44-54. (in Chinese)

[19] Luo X W, Zhang Z G, Zhao Z X, Chen B, Hu L, Wu X P. Design of DGPS navigation control system for Dongfanghong X-804 tractor. Transactions of the CSAE, 2009; 25(11): 139-145. (in Chinese)

[20] Erkan K, Erdal K, Herman R, Wouter S. Towards agrobots: Identification of the yaw dynamics and trajectory tracking of an autonomous tractor. Computers and Electronics in Agriculture, 2015; 115: 78-87.

[21] Li Y J, Zhao Z X, Huang P K, Guan W, Wu X P. Automatic Navigation System of Tractor Based on DGPS and Double Closed-loop Steering Control. Transactions of the CSAE, 2017; 48(2): 11-19. (in Chinese)

[22] Li Y J, Zhao Z X, Gao J W, Wu X P, Guan W. Agricultural automatic navigation system based on DGPS positioning and double closed-loop steering control. Research of Agricultural Modernization, 2016; 37(2): 387-394.

[23] Zang Y, Zang Y, Zhou Z Y, Gu X Y, Jiang R, Kong L X, et al. Design and anti-sway performance testing of pesticide tanks in spraying UAVs. Int J Agric \& Biol Eng, 2019; 12(1): 10-16.

[24] Zhou Z Y, Ming R, Zang Y, He X G, Luo X W, Lan Y B. Development status and countermeasures of agricultural aviation in China. Transactions of the CSAE, 2017; 33(20): 1-20. (in Chinese)

[25] Yu F H, Xu T Y, Du W, Ma H, Zhang G S, Chen C L. Radiative transfer models (RTMs) for field phenotyping inversion of rice based on UAV hyperspectral remote sensing. Int J Agric \& Biol Eng, 2017; 10(4): 150-157.

[26] Zhao D, Luo J, Sun Y P, Hong J Q, Zhang J. Design and experiment of navigation control system of automatic operation boat in river crab culture. Transactions of the CSAE, 2016; 32(11): 181-188. (in Chinese)

[27] Liu Z D, Lv Z Q, Zheng W X, Zhang W Z, Cheng X X. Design of obstacle avoidance controller for agricultural tractor based on ROS. Int $\mathrm{J}$ Agric \& Biol Eng, 2019; 12(6): 58-65.

[28] Hu J T, Gao L, Bai X P. Research progress on automatic navigation of agricultural machinery. Transactions of the CSAE, 2015; 31(10): 1-10. (in Chinese)

[29] Shamshiri R R, Weltzein C, Hameed I A, Yule I J, Grift T E, Balasundram $\mathrm{S} \mathrm{K}$, et al. Research and development in agricultural robotics: A perspective of digital farming. Int J Agric \& Biol Eng, 2018; 11(4): 1-14.

[30] Felix V H, Himmelsbach M, Hecker F, Andre M, Wuensche H J. Driving with tentaches: Integral structures for sensing and motion. Journal of Field Robotics, 2008; 25(9): 640-673.

[31] Velenis E, Frazzoli E, Tsiotras P. Steady-state Cornering equilibria and stabilization for a vehicle during extreme operating conditon. International Journal of Vehicle Autonomous Systems, 2010; 8(2-4): 217-241.

[32] Wang H, Wang G M, Luo X W, Zhang Z G, Gao Y, He J, et al. Path tracking control method of agricultural machine navigation based on aiming pursuit model. Transactions of the CSAE, 2019; 35(4): 11-19. (in Chinese)

[33] Yang J, Shi G S, Zhang W M, Zhao X, Dun H Y, Si J X. Path tracking algorithm of vehicles based on fuzzy hyperbolic tangent model. Transactions of the CSAE, 2017; 33(Z1): 78-84. (in Chinese)

[34] Shamshiri R R, Hameed I A, Pitonakova L, Weltzien C, Balasundram S K, Yule I J, et al. Simulation software and virtual environments for acceleration of agricultural robotics: Features highlights and performance comparison. Int J Agric \& Biol Eng, 2018; 11(4): 15-31.

[35] Costa C, Febbi P, Pallottino F, Cecchini M, Figorilli S, Antonucci F, et al Stereovision system for estimating tractors and agricultural machines transit area under orchards canopy. Int J Agric \& Biol Eng, 2019; 12(1): $1-5$.

[36] Liu Z D, Lv Z Q, Zhang W Z. Design and experiment of path tracking of agricultural vehicle. IAEJ, 2018; 27(4): 44-52. 